\title{
Antibioticoterapia Profilática em Obstetrícia: Comparação entre Esquemas
}

\author{
Prophylactic Antibiotic Treatment in Obstetrics: \\ Comparison of regimens
}

Heraldo Francisco Costa, Ivete de Ávila, Manuel Maurício Gonçalves

\section{RESUMO}

Objetivos: avaliar a eficiência de vários esquemas de antibioterapia profilática no parto na prevenção da infecção puerperal.

Métodos: segundo a via de parto (vaginal ou abdominal) e conforme a presença ou não de um ou mais fatores de risco para infecção puerperal, as pacientes foram distribuidas entre os grupos de baixo, médio e alto risco para a infecção puerperal. Foram incluídas 2.263 pacientes no periodo de março de 1994 a junho de 1997.

Resultados: a incidência de infecção puerperal variou entre os grupos. Foi de 3,1\% no grupo de baixo risco, em que nenhum antibiótico foi administrado e de 8, 5\% no grupo de alto risco, no qual todas as pacientes receberam cefalotina $1 \mathrm{~g} \mathrm{EV}$ em três doses, com intervalo de seis horas entre as doses. No grupo de médio risco a taxa de infecção puerperal foi de 5,3\% entre as pacientes que receberam cefoxitina $1 \mathrm{~g} \mathrm{EV}$ em três doses; 5,0\% entre as usuárias de cefalotina $1 \mathrm{~g} \mathrm{EV}$ em três doses; 4,0\% quando se utilizou a cefoxitina em dose única $e$ $3,4 \%$ quando utilizou-se cefalotina em dose única.

Conclusões: no grupo de baixo risco é desnecessária a antibioticoterapia profilática. A cefalosporina de $2^{a}$ geração (cefoxitina) teve eficácia semelhante a de $1^{a}$ geração (cefalotina) na prevenção a infecção puerperal, independente da posologia utilizada. A cefalotina parece ser eficaz na prevenção da infecção puerperal no grupo de alto risco.

PALAVRAS-CHAVE: Infecção puerperal. Antibioticoprofilaxia. Cesariana. Parto vaginal. Amniorrexe prematura.

Introdução

Hospital dos Servidores do Estado de Minas Gerais - IPSEMG Belo Horizonte - Minas Gerais

Correspondência:

Manoel Maurício Gonçalves

Rua Estevão Pinto, 350, apto. 1001

30210-000 - Belo Horizonte - MG
A incidência de infecção puerperal varia com o tipo de população estudada, com o uso ou não da antibioticoterapia profilática, a via de parto (vaginal ou abdominal) e as condições nas quais 
este se desenrolou (eletivo ou de emergência) ${ }^{1,8}$. Este processo mórbido é responsável por 35\% dos óbitos maternos em países desenvolvidos e, provavelmente, é a principal causa de morte materna no Brasil ${ }^{10}$.

A incidência crescente de cesarianas tem contribuído nas últimas décadas, para o aumento das taxas de infecção puerperal ${ }^{5,11}$. De fato esta está associada a maior incidência de morbidade infecciosa pós-operatória quando comparada ao parto normal, com taxa abaixo de $10 \%^{2,4}$. A complicação mais comum após cesariana é a endometrite, variando sua incidência de 5 a 85\% conforme a população em estudo ${ }^{5}$.

As taxas de endometrite pós-cesariana podem se reduzidas em aproximadamente $50 \%$ com o uso da antibioticoterapia profilática ${ }^{7}$. No entanto questiona-se se a profilaxia deveria ser realizada em todas as mulheres, qual a droga mais apropriada e quando iniciar a sua administração e a duração do uso. Há tendência atual ao uso das cefalosporinas, iniciando-se a sua administração no pré-operatório ou após o clampeamento do cordão umbilical, em dose única ou no máximo por doze horas de pós-operatório ${ }^{12}$.

Aparentemente não há diferença significativa na eficácia das cefalosporinas de primeira ou terceira geração ${ }^{3}$. A administração do antibiótico após o clampeamento do cordão umbilical parece não alterar sua capacidade em prevenir a infecção puerperal $^{5,9,12}$. Estudos comparando uma mesma droga usada em doses diferentes, isto é, dose única versus múltiplas doses, não mostram diferença na eficácia de um ou outro regime ${ }^{13}$.

Com base nestes dados, este estudo foi desenvolvido com o objetivo de comparar a eficácia de quatro esquemas de cefalosporinas usadas profilaticamente no parto (para prevenção da infecção puerperal) em grupos de pacientes divididas conforme o risco para apresentarem infecção puerperal.

\section{Pacientes e Métodos}

Trata-se de estudo prospectivo e aleatório cujos dados foram obtidos no período de março de 1994 a junho de 1997. Foram incluídas 2.263 pacientes, distribuídas entre os grupos de baixo, médio e alto risco para o desenvolvimento de infecção puerperal, conforme a via de parto e a presença ou não de um ou mais fatores de risco (Tabela 1).

Baseado na literatura consideramos os fatores de riscos enumerados na Tabela 2 .
Tabela 1 - Grupos de risco para a infecção puerperal e profilaxia empregada

\begin{tabular}{|c|c|c|c|}
\hline Grupo & Risco & Conceito & Antibioticoterapia \\
\hline \multirow[t]{2}{*}{1} & Baixo & $\begin{array}{l}\text { Parto vaginal } \\
\text { sem fator de risco }\end{array}$ & Ausente \\
\hline & & & $\begin{array}{l}1 \text { - Cefalotina } 1 \mathrm{~g} \mathrm{EV} \\
\text { dose única }\end{array}$ \\
\hline \multirow[t]{3}{*}{2} & Médio & $\begin{array}{l}\text { Cesariana sem } \\
\text { fator de risco ou }\end{array}$ & $\begin{array}{l}2 \text { - Cefoxitina } 1 \mathrm{~g} E V \\
\text { dose única }\end{array}$ \\
\hline & & $\begin{array}{l}\text { parto vaginal } \\
\text { com fator de risco }\end{array}$ & $\begin{array}{l}3 \text { - Cefalotina } 1 \mathrm{~g} \text { EV } 3 \\
\text { doses }\end{array}$ \\
\hline & & & $\begin{array}{l}4 \text { - Cefoxitina } 1 \mathrm{~g} \mathrm{EV} 3 \\
\text { doses }\end{array}$ \\
\hline 3 & Alto & $\begin{array}{l}\text { Cesariana com } \\
\text { fator de risco }\end{array}$ & $\begin{array}{l}\text { Cefalotina } \\
\text { doses }\end{array}$ \\
\hline
\end{tabular}

Tabela 2 - Fatores de risco para infecção puerperal

Bolsa rota há 6 horas ou mais

Trabalho de parto com duração de 8 horas ou mais

Toques vaginais em número de 7 ou mais

Uso de sonda vesical

Fórceps

Manobras de extração fetal ou placentária

Laceração de colo uterino ou vagina

Laceração do reto

Hemorragia pré ou intra-parto

Anemia (mucosa hipocorada ou hemoglobina inferior a $11 \mathrm{~g} \%$ )

Obesidade

Diabetes mellitus

Estado geral comprometido

Baixo nível socio-econômico

Resolução de urgência

Doenças associadas

O nosso protocolo previa que:

Nenhuma paciente do grupo de baixo risco receberia antibióticoterapia profilática;

As pacientes do grupo de médio risco receberiam aleatoriamente um dos seguintes esquemas de antibioticoterapia profilática, iniciados sempre após o clampeamento do cordão umbilical;

- cefalotina $1 \mathrm{~g} \mathrm{EV}$, dose única intraparto;

- cefalotina $1 \mathrm{~g}$ EV de seis em seis horas, três doses;

- cefoxitina $1 \mathrm{~g} \mathrm{EV}$, dose única intraparto;

- cefoxitina $1 \mathrm{~g}$ EV de seis em seis horas, três doses

Todas as pacientes do grupo de alto risco receberiam $1 \mathrm{~g}$ de cefalotina $\mathrm{EV}$ com intervalos de seis horas, num total de três doses, sendo a 
primeira, logo após o clampeamento do cordão umbilical.

Utilizou-se para comparar os três grupos de risco e os quatro esquemas de antibiótico, em relação à incidência de infecção puerperal o teste do $\chi^{2}$. Este teste é indicado quando o objetivo é comparar proporções entre grupos. Nas situações em que se observaram diferenças significativas, utilizou-se a partição de tabelas para determinar as diferenças existentes entre os grupos. E, avaliouse a relação existente por meio da razão das chances $(\mathrm{OR})^{13,21,22,26}$.

Em todos os casos estudados foram obedecidas as normas padronizadas em relação a antissepsia, técnica cirúrgica e curativo pós-operatório.

O estudo foi aprovado pela Comissão de Ética do Hospital e pela Comissão de Controle de Infecção Hospitalar.

\section{Resultados}

Das 2.263 pacientes incluídas neste estudo, 120 desenvolveram infecção puerperal (5, 3 \%).

No grupo de baixo risco, com 552 pacientes, 17 evoluíram com infecção (3,1\%). Foram registrados cinco casos de endometrite, seis casos de infecção do trato urinário e sete casos de infecção na episiorrafia.

Já o grupo de alto risco, contou com 683 pacientes das quais 58 apresentaram infecção puerperal (8,5\%). O tipo mais comum de infecção foi a de parede abdominal seguido pela endometrite (Tabela 3) .

Tabela 3 - Incidência de infeção puerperal conforme o grupo de risco e comparação com os dados de $\mathrm{Gibbs}^{17}$

\begin{tabular}{ccccc}
$\begin{array}{c}\text { Grupo de } \\
\text { risco }\end{array}$ & $\begin{array}{c}\mathbf{N}^{\mathbf{0}} \text { total } \\
\text { pacientes }\end{array}$ & $\begin{array}{c}\text { Infecção } \\
\text { Puerperal }\end{array}$ & Incidência (\%) \\
\hline $\mathrm{n}$ & $\mathrm{n}$ & IPSEMG (1997) & GIBBS
\end{tabular}

\begin{tabular}{lrrcc}
\hline Baixo & 552 & 17 & 3,1 & 1 a 3 \\
Médio & 1.028 & 45 & 4,4 & 3 a 10 \\
Alto & 683 & 58 & 8,4 & 10 a 85 \\
\hline TOTAL & 2.263 & 120 & 5,3 & -
\end{tabular}

Das 1.028 pacientes do grupo de médio risco, 45 desenvolveram infecção puerperal $(4,4 \%)$. Neste grupo a incidência de infecção não variou conforme o esquema de antibiótico utilizado (Tabela 4).
Tabela 4 - Incidência de infeção puerperal nas pacientes do grupo de médio risco segundo o esquema posológico utilizado

\begin{tabular}{|c|c|c|c|}
\hline \multirow[t]{2}{*}{ Esquema } & \multirow[b]{2}{*}{$\mathrm{n}$} & \multicolumn{2}{|c|}{ Pacientes com infecção } \\
\hline & & $\mathrm{n}$ & $\%$ \\
\hline $\begin{array}{c}\text { Cefoxitina } 1 \mathrm{~g} \mathrm{EV} \text { dose } \\
\text { única }\end{array}$ & 276 & 11 & 4,0 \\
\hline $\begin{array}{c}\text { Cefalotina } 1 \mathrm{~g} \text { EV dose } \\
\text { única }\end{array}$ & 291 & 10 & 3,4 \\
\hline $\begin{array}{c}\text { Cefoxitina } 1 \mathrm{~g} \text { EV três } \\
\text { doses }\end{array}$ & 207 & 11 & 5,3 \\
\hline $\begin{array}{c}\text { Cefoxitina } 1 \mathrm{~g} \text { EV três } \\
\text { doses }\end{array}$ & 254 & 13 & 5,1 \\
\hline TOTAL & 1.028 & 45 & 4,4 \\
\hline
\end{tabular}

Analisando os 120 casos com infecção ocorridos após o uso profilático de antibiótico, observamos que estes predominaram na faixa etária dos 21 aos 30 anos e foram mais freqüentes após cesariana $(7,9 \%)$ do que após parto vaginal $(3,2 \%)$.

Os principais fatores de risco que se associaram a infecção puerperal foram o uso de sonda vesical, o número de toques vaginais durante o trabalho de parto, rotura das membranas por seis horas ou mais, e o uso de fórceps.

A Tabela 5 mostra a percentagem global de cesariana e em cada grupo de risco e as respectivas taxas de infecção puerperal.

Tabela 5 - Percentagem global de cesariana e por grupo de risco e respectivas taxas de infecção

\begin{tabular}{lcc} 
Grupo de risco & $\begin{array}{c}\text { Incidência de } \\
\text { cesariana } \\
(\boldsymbol{\%})\end{array}$ & $\begin{array}{c}\text { Incidência de } \\
\text { infecção puerperal } \\
(\%)\end{array}$ \\
\hline Baixo & - & - \\
Médio & 31,7 & 6,7 \\
Alto & 100,0 & 8,5 \\
\hline TOTAL & 44,6 & 7,9
\end{tabular}

Como pode ser observado na Tabela $6 \mathrm{e}$ Figura 1, a incidência de infecção puerperal no grupo de baixo risco foi de $3,1 \%$, no grupo de médio risco foi de $4,4 \%$ e no grupo de alto risco $8,5 \%$. Além disso, os resultados mostraram que não existe 
diferença significativa entre os grupos de baixo e médio risco, mas ambos apresentaram uma incidência de infecção puerperal significativamente inferior $(\mathrm{p}<0,001)$ à observada o grupo de alto risco. Sendo que uma paciente do grupo de alto risco têm 2,3 vezes mais chance de apresentar uma infecção puerperal do que uma paciente dos grupos de baixo ou médio risco.

Tabela 6 - Avaliação da incidência de infecção puerperal conforme o grupo de risco

Grupo de risco Infecção

\begin{tabular}{lrcccr} 
& \multicolumn{2}{c}{ Sim } & & Não & Total \\
& $\mathrm{n}$ & $\%$ & $\mathrm{n}$ & $\%$ & \\
\hline Baixo & 17 & 3,1 & 535 & 96,9 & $\mathbf{5 5 2}$ \\
Médio & 45 & 4,4 & 983 & 95,6 & $\mathbf{1 . 0 2 8}$ \\
Alto & 58 & 8,5 & 625 & 91,5 & $\mathbf{6 8 3}$ \\
\hline
\end{tabular}

$\mathrm{p}<0,001$ teste do $\chi^{2}$

$\mathrm{OR}=2,3$ para comparação entre o grupo de alto risco com os demais

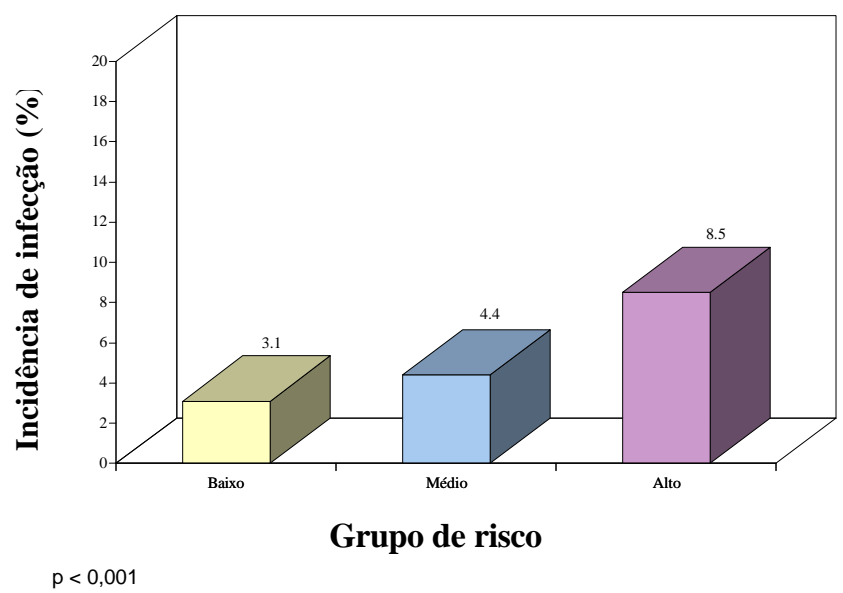

Figura 1 - Incidência de infecção puerperal conforme o grupo de risco puerperal no grupo de médio risco, considerando os quatro esquemas de antibiótico utilizados, não se verificou diferenças significativas sendo que a incidência variou de 3,4\% a 5,3\% (Tabela 7 e Figura 2). Desta forma, pode-se dizer que, em termos de eficácia, os quatro esquemas são semelhantes.
Tabela 7 - Avaliação da incidência de infecção puerperal no grupo de médio risco conforme o esquema de antibióticos

Infecção

\begin{tabular}{|c|c|c|c|c|c|}
\hline \multirow[t]{2}{*}{ Esquema de antibiótico } & \multicolumn{2}{|c|}{ Sim } & \multicolumn{2}{|c|}{ Não } & \multirow[t]{2}{*}{ Total } \\
\hline & $\mathrm{n}$ & $\%$ & $\mathrm{n}$ & $\%$ & \\
\hline $\begin{array}{c}\text { Cefoxitina } 1 \mathrm{~g} \mathrm{EV} \\
\text { dose única }\left(\mathrm{M}_{1}\right)\end{array}$ & 11 & 4,0 & 265 & 96,0 & 276 \\
\hline $\begin{array}{c}\text { Cefoxitina } 1 \mathrm{~g} \mathrm{EV} \\
\text { dose única }\left(\mathrm{K}_{1}\right)\end{array}$ & 10 & 3,4 & 281 & 96,6 & 291 \\
\hline $\begin{array}{c}\text { Cefoxitina } 1 \mathrm{~g} \mathrm{EV} \\
\text { três doses }\left(\mathrm{M}_{3}\right)\end{array}$ & 11 & 5,3 & 196 & 94,7 & 207 \\
\hline $\begin{array}{c}\text { Cefalotina } 1 \mathrm{~g} \mathrm{EV} \\
\text { três doses }\left(\mathrm{K}_{3}\right)\end{array}$ & 13 & 5,1 & 241 & 94,9 & 254 \\
\hline
\end{tabular}

Nota: $\mathrm{p}=0,686 \chi^{2}$

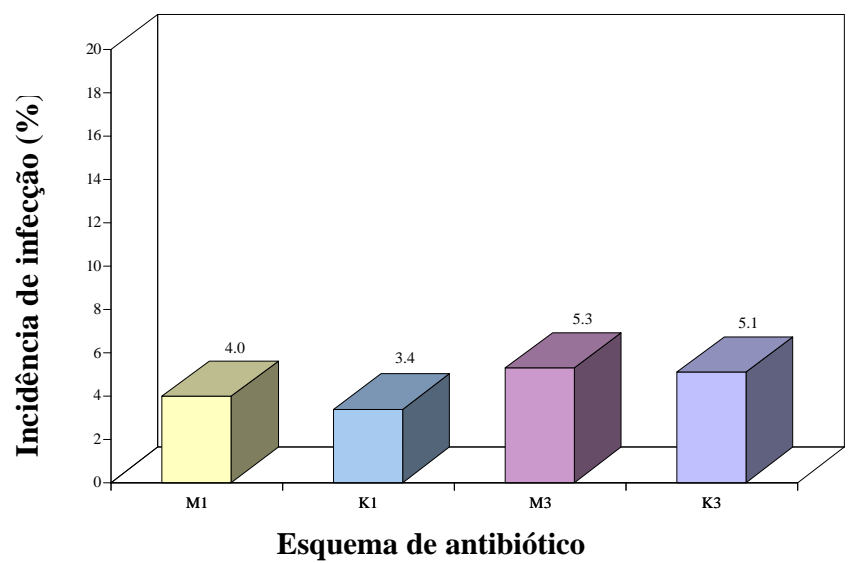

$p=0,686$

$\mathrm{M}_{1}$ : cefoxitina $1 \mathrm{~g} E V$ dose única; $K_{1}$ : cefalotina $1 \mathrm{~g} E V$ dose única; $\mathrm{M}_{3}$ : cefoxitina $1 \mathrm{~g} E \mathrm{EV}$ trềs doses; $K_{3}$ : cefalotina $1 \mathrm{~g} E \mathrm{E}$ três doses.

Figura 2 - Incidência de infecção puerperal no grupo de médio risco conforme o esquema de antibióticos

\section{Discussão}

A antibioticoterapia profilática pode ser definida como a administração de um agente antimicrobiano de toxicidade mínima por um período curto de tempo, administrado a pacientes sem infecções e com risco considerável para infecção, tendo como objetivo reduzir o risco de 
infecção pós-operatória ou para reduzir a contaminação no campo operatório ${ }^{15}$, 20, 25, 28.

Dentre os vários fatores de risco citados na literatura estão os seguintes: baixo nível sócioeconômico ${ }^{6}$, longa duração de rotura das membranas $^{12}$, longa duração do trabalho de parto ${ }^{12}$, excessivo número de exames vaginais ${ }^{12}$, longa duração de monitorização fetal interna ${ }^{12}$, anemia pré e pós-operatória ${ }^{18}$, fórceps ${ }^{17}$, nuliparidade ${ }^{18}$, inexperiência do cirurgião ${ }^{12}$, obesidade ${ }^{19}$, violação das técnicas de esterilização ${ }^{19}$, longo tempo cirúrgico: maior que $1 \mathrm{~h}^{19}$, cesariana de urgência ${ }^{19}$, diabetes ${ }^{10}$, drenos superficiais ${ }^{12}$.

A maioria dos estudos demonstram que nas pacientes de baixo nível sócio-econômico, independente da raça, a infecção puerperal tem uma morbidade maior. Estas pacientes apresentam maior incidência de desnutrição, anemia, além de hábitos inadequados de higiene e também menor assistência pré-natal ${ }^{17,27}$.

Em nosso estudo, o uso de sonda vesical foi o fator de risco mais associado à infecção puerperal, seguido dos fatores de risco apresentados pelos autores anteriormente citados. É importante a realização de urocultura após o uso de sonda vesical, uma vez que os principais germes encontrados, i.e., E. coli e enterococos, podem ser causa de endometrite ${ }^{9}$.

As contrações uterinas durante o trabalho de parto e a rotura das membranas favorecem a colonização bacteriana da cavidade uterina (a primeira por mecanismos de sucção e a segunda por fenômenos locais) ${ }^{14}$.

Entre as pacientes que evoluem para cesariana, a obesidade não tem sido considerada como fator de risco consistente, mas é o principal fator de risco para infecção da ferida operatória ${ }^{16}$. A combinação de extração manual da placenta e a exteriorização uterina aumentam significativamente a incidência de endometrite pós-cesariana em relação à dequitadura espontânea da placenta e à não exteriorização uterina durante a histerorrafia ${ }^{23}$.

O fórceps pode contribuir para a infecção em razão das lacerações, pois as áreas cruentas funcionam como meio de cultura. Todavia, as pacientes que são submetidas ao fórceps, podem já ser portadoras de outros fatores de risco ${ }^{17}$.

Pacientes diabéticas insulino-dependentes apresentam maior índice de infecção póscesariana que as não diabéticas e dentre as diabéticas, tinham maior risco as com controle glicêmico deficiente ${ }^{10}$.

Gibbs $^{17}$ identificou quatro grupos de risco para infecção puerperal, de acordo com as características clínicas (Tabela 8).

Há consenso de que não há vantagens em usar antibiótico profilático no parto vaginal, mesmo considerando-se que fatores de risco como baixo nível sócio-econômico e antissepsia precária estejam presentes ${ }^{8}$. Tem-se discutido exaustivamente, qual o antibiótico ideal para a profilaxia da infecção na operação cesariana, existindo uma gama muito extensa de antibióticos para este fim, em doses e esquemas diferentes ${ }^{24}$.

No grupo de baixo risco no qual não se empregou antibioticoterapia profilática, comprovou-se não haver necessidade da mesma.

No grupo de médio risco, não houve diferenças estatisticamente significativas, a incidência de infecção puerperal não apresentou quando se compara os antibióticos utilizados.

A cefoxitina e a cefalotina mostraram eficácia semelhante em prevenir a infecção puerperal no grupo de médio risco, tanto quando usadas em dose única, como em três doses.

No grupo de alto risco, a cefalotina sugere ser eficaz em prevenir a infecção puerperal quando administrada em três doses.

Tabela 8 - Grupos de risco para infecção puerperal (Gibbs $\left.{ }^{17}\right)$

\begin{tabular}{|c|c|}
\hline Grupo de Risco & Fatores de Risco \\
\hline Muito alto & Operação cesariana após trabalho de parto e \\
\hline$(40-85 \%) *$ & $\begin{array}{l}\text { amniorrexe > 6-12 horas, com múltiplos } \\
\text { exames vaginais em mulheres indigentes }\end{array}$ \\
\hline Alto & Operação cesariana após trabalho de parto e \\
\hline$(10-40 \%) *$ & $\begin{array}{l}\text { amniorrexe }<6 \text { horas ou cesariana eletiva em } \\
\text { mulheres indigentes }\end{array}$ \\
\hline Moderado & $\begin{array}{l}\text { Operação cesariana eletiva em mulheres } \\
\text { não-indigentes }\end{array}$ \\
\hline$(3-10 \%) *$ & $\begin{array}{l}\text { Parto vaginal e amniorrexe prolongada ou com } \\
\text { grande traumatismo }\end{array}$ \\
\hline Baixo & Parto vaginal não-complicado \\
\hline$(1-3 \%) *$ & \\
\hline
\end{tabular}

* incidência de infecção puerperal.

\section{SUMMARY}

Purpose: to evaluate the efficacy of four antibiotic regimens in puerperal infection prophylaxis.

Patients and Methods: According to vaginal or abdominal delivery and risk the presence or not of factors for puerperal infection, the patients were allocated to groups of low, medium and high risk for its development. Between March 
1994 and June 1997 2,263 patients were evaluated.

Results: the incidence of puerperal infection was different in each group. It was $3.1 \%$ in the low risk group, where no antibiotic was given, and $8.5 \%$ in the high risk group where all patients received three doses of $1 \mathrm{~g} E V$ cefalotin at sixhour intervals. In the medium risk group, the incidence of puerperal infection was $5.3 \%$ for the patients who used three doses of $1 \mathrm{~g}$ EV cefoxitin; $5.1 \%$ for those who used three doses of $1 \mathrm{~g}$ EV cefalotin; 4.0\% when a single cefoxitin dose was used and $3.4 \%$ when a single cefalotin dose was used.

Conclusions: it is not necessary to use prophylactic antibiotic therapy in low risk patients and the first generation cephalosporins (cefalotin) are as efficacious as the second generation cephalosporins (cefoxitin) to prevent puerperal infection, independent of the applied dosage. Cefalotin seems to be effective in preventing puerperal infection in patients at high risk.

KEY WORDS: Puerperal infection. Antibiotic prophylaxis. Rupture of membranes, premature. Delivery, vaginal. Cesarean section.

\section{Referências}

1.Adair CD, Ernest JM, Sanchez-Ramos L, Burrus DR, Boles ML, Veille JC. Meconium-stained amniotic fluid-associated infectious morbidity: a randomized, double-blind trial of ampicillinsulbactam prophylaxis. Obstet Gynecol 1996; 88: 216-20.

2.Barros ACSD, Cha SC, Zugaib M. Roteiro para antibioticoterapia profilática em obstetrícia. Rev Paul Med, 1988; 106: 343-9.

3.Bates T, Siller G, Crathern BC, Bradley SP, Zlotnik $\mathrm{RD}$, Couch $\mathrm{C}$ et al. Timing of prophylactic antibiotics in abdominal surgery: trial of a preoperative versus an intra-operative first dose. Br J Surg, 1989; 76: 52-6.

4.Blanco JD, Gibbs RS, Krebs LF, Castaneda YS. The association between the absence of amniotic fluid bacterial inhibitory activity and intraamniotic infections. Am J Obstet Gynecol 1982; 143: 749 -55.

5.Chan AC, Leung AK, Chin RK, Chang AM. Single dose prophylactic antibiotics in caesarean sections. Aust N Z J Obstet Gynaecol 1989; 29: 107-9.

6.Chang PL, Newton ER. Predictors of antibiotic prophylactic failure in post-cesarean endometritis. Obstet Gynecol 1992; 80:117-22.

7.Chapman S, Duff P. Incidence of chorioamnionitis in patients with meconium-stained fluid. Infect Dis Obstet Gynecol 1995; 2: 210-2.

8.Classen DC, Evans RS, Pestotnik SL, Horn SD,
Menlove RL, Burke JP. The timing of prophylactic administration of antibiotics and the risk of surgical wound infection. N Engl J Med 1992; 326: 281-6.

9.Desjardins C, Diallo HO, Audet-Lapointe P, Harel F. Retrospective study of post-cesarean endometritis 1992-1993, Notre Dame Hospital, Montreal, Canada. J Gynecol Obstet Biol Reprod, 1996; 25:419-23.

10.Diamond MP, Entman SS, Salyer SL, Vaughn WK, Boehm FH. Increased risk of endometritis and wound infection after cesarean section in insulindependent diabetic women. Am J obstet Gynecol1986; 155: 297-300.

11.Duff P, Gibbs RS, St. Clair PJ, Weinberg LC. Correlation of laboratory and clinical criteria in the prediction of postcesarean endomyometritis. Obstet Gynecol 1984; 63:781-6.

12.Emmons SL, Krohn M, Jackson M, Eschenbach DA. Development of wound infections among women undergoing cesarean section. Obstet Gynecol 1988; 72:559-64.

13.Everitt BS. The Analysis of Contingency Tables. London: Chapman and Hall, 1989.

14.Faro S. Antibiotic prophylaxis. Obstet Gynecol Clin North Am 1989; 16:279-89.

15.Faro S, Martens M, Hammill H, Phillips LE, Smith D, Riddle G. Ticarcilin/Clavulanic acid versus clindamycin and gentamicin in the treatment of post-cesaren endometritis following antibiotic prophylaxis. Obstet Gynecol 1989;73:808-12.

16.Faro S, Martens MG, Hammill HÁ, Riddle G, Tortolero G. Antibiotic prophylaxis: Is there a difference? Am J Obstet Gynecol 1990; 162:900-7.

17.Gibbs RS. Clinical risk factors for puerperal infection. Obstet Gynecol 1980; 55:178-84S.

18.Guldholt I, Espersen T. Maternal febrile morbidity after cesarean section. Acta Obstet Gynecol Scand 1987; 66:675-9.

19.Hemsell DL. Prophylactic antibiotics in gynecologic and obstetric surgery. Rev Infect Dis 1991; 13: S821-41 (Review).

20.Herbst AL, Mercer LJ. Overview of therapeutic and prophylactic antibiotics in obstetrics and gynecology. J Reprod Med 1988; 33:144-8.

21.Johnson R, Bhattacharyya G. Statistics Principles and Methods. New York: John Wiley \& Sons, 1986.

22.Jonsson S, Pulkkinen MO. Mastitis today: incidence, prevention and treatment. Ann Chir Gynaecol Suppl 1994; 208:84-7.

23-Magann EF, Washburne JF, Harris RL, Bass JD, Duff, WP, Morrison JC. Infectious morbidity, operative blood loss, and length of the operative procedure after cesarean delivery by method of placental removal and site of uterine repair. 
J Am Coll Surg 1995; 181:517-20.

24. Remigio Neto J, Falcone R, Freire S, Lima JC. Antibioticoprofilaxia da infecção pós-cesárea com uso do cefoxitina. Rev Ginecol Obstet 1995; 6:36-41.

25.Ribeiro CC, Silva LGP, Amim Júnior J, Jürgensen $\mathrm{CA}$, Montenegro CAB. Antibioticoprofilaxia na morbidade febril pós-cesariana. J Bras Ginecol 1989; 99:135-42.
26.Sas Institute Inc. SAS User's Guide Statistic Version 5. Cary NC: SAS Institute Inc; 1985.

27.Silva CHA, Soares CS, Paulino I. Estudo prospectivo e randomizado do uso de antimicrobiano profilático em cesarianas. Rev Bras Profiss Contr Infec Hosp 1993; 2:4-6.

28.Stein GE. Patient costs for prophylaxis and treatment of obstetric and gynecologic surgical infections. Am J Obstet Gynecol 1991; 164: 1377-80. 